\title{
Criteria for objectivity and non-objectivity in linguistics
}

\author{
Galina Matveeva ${ }^{1, *}$, Georgy Myasischev ${ }^{1}$, Olga Gaibaryan $^{2}$ and Elena Shirina ${ }^{2}$ \\ ${ }^{1}$ Don State Technical University, Gagarin,1, Rostov-on-Don, 344003, Russia \\ ${ }^{2}$ South Federal University, 105/42 Bolshaya Sadovaya Str., Rostov-on-Don, 344006, Russia
}

\begin{abstract}
This article is dedicated to studying a few problems of objective and non-objective approaches towards linguistic research and their impact on the educational potential of higher school. The article also presents the following: a review of the attitudes of the learner towards the information about liberal arts; a review of relevant internet resources and an analysis of the level of their objectiveness; the key indices of the non-objective approach towards linguistics; an example of learners' responses to a spectrum of sources of knowledge about linguistics and an example of objective linguistic approach towards studying language and speech, We are offering a detailed theoretical description of the model of the linguistic persona, pragmalinguistic experiments, links to a few more successful works in the field, details of an experiment based on some pragmalinguistic methods and its results. The method applied is based on comparing among a number of specific syntactic unities and applying some grammar forms all along the documentary body under study.
\end{abstract}

\section{Introduction}

Contemporary philology is making an effort to conceptualize anew the criterion of objectivity. Research papers are crammed with discussions on the agenda of now the objective and non-objective may correlate. There are cases in social sciences and liberal arts where it is especially cumbersome to differentiate between them, should the researchers at that stick to postmodern methodologies $[1,2,9]$. The concept of leveling a personal opinion and objective knowledge, proclaimed by some insistent authors, makes philologists face the question of whether any objective knowledge is possible at all? Or is it liberal arts, linguistics in particular, are nothing but a bundle of opinions?

The article discusses the questions of determining the limits of objective and nonobjective types of knowledge in terms of teaching linguistic subjects at higher school.

Keeping this in mind, we are offering a presentation of a non-objective approach to the issues of linguistics as presented in the contemporary mass communication means and how the learner may be impacted through that.

The representation of the objective methods of linguistic analysis is given based on an example of the concrete methodology of objective pragmalinguistic experimenting and simulation.

\footnotetext{
* Correspondibg author: oc41@mail.ru
} 


\section{Materials and method}

One of the key issues of contemporary learning lies in competition between the knowledge taught at higher school and the one found in some popular information sources, primarily those of the WWW. It is not a secret that today's student is a rather mobile, outgoing person spending a great deal of time in the Internet virtual space [27]. The natural drive for knowledge urges them to stumble upon a variety of these sources and gain quite a different understanding of the objective world around. Quite often, such knowledge may be quite false.

Moreover, lacking professional personal experience and being full of protests against the "burden of common sense" they can easily get caught in the web of extreme concepts and doctrines. While in the times where virtual communication never existed or was not spread widely enough (the 2000's), public information among the young and those interested in liberal arts was supplied by qualified popular science promoters via popular science newspapers, magazines, and brochures, nowadays the ultimate opinion may be shared by any Internet user.

The authors of the article never aim at studying the reasons that make those lacking sound knowledge plunge into educational activities. Instead, we are going to just touch upon a few examples of how non-objective information sources may be in demand among the young.

While looking into the questions of the Internet dilettantism, which is wearing a mask of scientific education, we have performed an analysis of a number of popular sites positioned as humanitarian (literary). The criterion of popularity here was the number of subscribers, coming up from a thousand to ten thousand people, according to the site statistics.

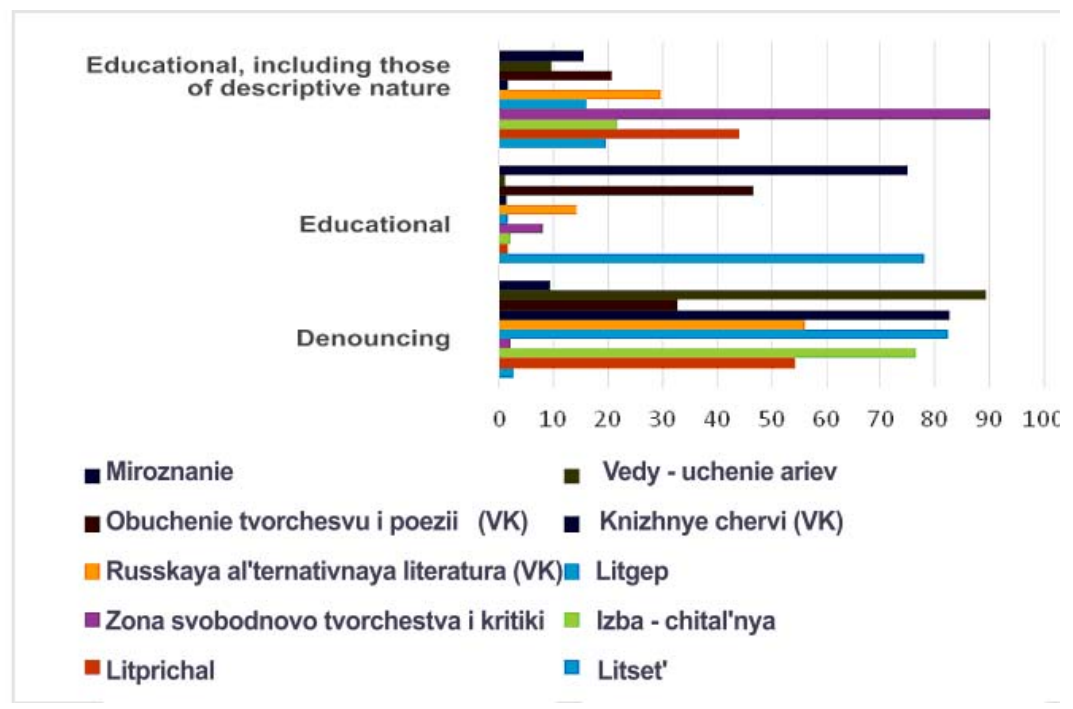

Fig. 1. Distribution of articles along generalized enlarged topic scale (from 5 to $100 \%$ of all articles).

Diagram 1 show the distribution of the topics of articles, according to the statistical data of the sites and communities in the social network VKontakte (marked as "VK").

We have never estimated how the contents of articles were justifiable from the point of view of science, but only looked into the presence and direction of publications of educational and promotional nature in the field of literature. The results obtained snow that these questions are in public demand and often discussed by Internet communities.

To analyze the level of demand in the alternative sources of knowledge among the undergraduates of the Don State Technical University, we performed a questionnaire (Diagram 2) 
The public space information sources under study were estimated by the participants of the query basing on the levels of demand, usability, and resource intensity (i.e. amount of a source user's satisfaction about information content as percentage of the number of users)

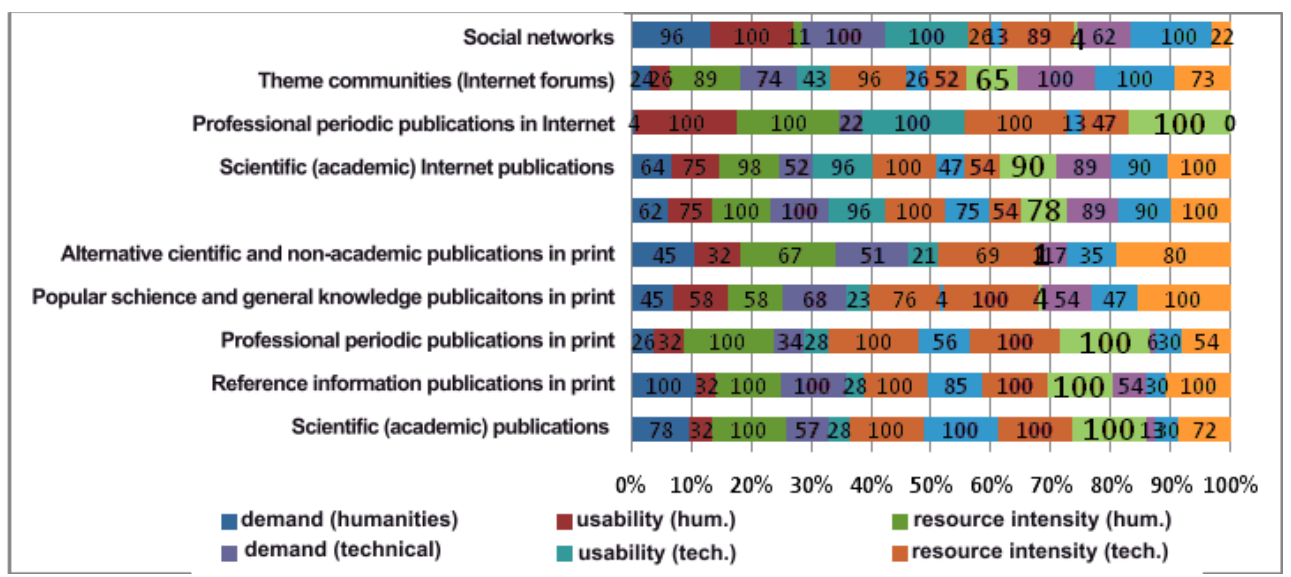

Fig. 2. Analysis of Choice of WWW Information Sources.

As we may see in the diagram, academic and reference literature seems of high demand among the students and staff. The high level of trust in alternative scientific and nonacademic literature has been shown by the students, which, in view of the low interest in professional publications, speaks both of a certain amount of skepticism towards scientific knowledge and low scientific and practical experience. High results are shown by the questionnaire in terms of looking for scientific and reference data in social networks, which speaks of inseparability of sources and also certain trust in the printed word, irrespective of where it comes from. Thus, the profile academic medium is perceived unfavorably, i.e. regarded poor for realization in the eyes of the student.

Those listed in the questionnaire resources were studied from the angle of how their contents matched the criteria of the objectivity of scientific knowledge $[3,7,13]$.

We discovered that, in view of the presented resources, among other publication, there were also articles on linguistic and cultural aspects. Their authors speak of the high scientific and objective value of their publications. However, their contents lie aside from the objective approach towards linguistics in particular and science in general. Like, the site Litset presents a cycle of publications from the user nicknamed Byr. One of their publications is entitled "The Grammar and Technology of PoDskal'zyvaniya". The author aims at denouncing the conspiratorial nature of "scientific snobs" trying to prove that, contrary to spelling rules, it is permissible to use the verb form "podskol'znutsya" instead of "poskol'znutsya". It should be noted that the author discusses the grammatical form of the verb "podskil'znutsya" by highlighting the importance of the prefix "pod-". The peculiarities of the author's way of proof are evident all along the article. In testimony, the author quotes the body of rules of some literary monuments of the 19th century by writing that "It is at least strange to consider the verb "podskol'znutsya" nonliterary and colloquial. Authors actively use the word not only in the characters' direct speech, where you may freely use everyday colloquialisms, but also in the author's discourse" (http://litset.ru/publ/7-1-0-59553. However, the author conceals the fact that contemporary writers make us of "podskil'znutsya" not for the purpose of describing the speech culture of the character, nor the author present a definition to the "author's discourse".

In conclusion, "Vyr" suggests that one should not differentiate between the existing literary "poskol'znutsya" and "podskol'znutsya" by just writing "Hence, let us shape up a new concept of the verb "podskol'znutsya' meaning 'temporarily lose balance on a slippery spot 
but straighten back and avoid falling down" (prefix "pod-" meaning an incomplete snort action)". (http://litset.ru/publ/7-1-0-59553)

The same author of the article "Lozhili and Will Continue Lozhit' "takes up a leading role in introducing the verb "lozhit' " to the normal literary language by writing: "Why the reflexive verb "lozhit'sya" was "allowed" is a mystery. As early as in the 18th century, it was evident to every educated person that one may and should "ukladyvatsya", while "lozhitsya" was rather indecent! But moralists lost the moment, merely blundered before it was too late. It should be justifiable to return the notional verb "lashed"' to the Russian cultural vocabulary as correct, meaningful, and clear-cut. No logical contradictions can be found here." (http://litset.ru/publ/7-1-0-60394)

Despite being sorry about the above "scientific findings" caused by to the author's unwillinness to learn the history of the language and it functioning, such publications attract a bunch of positive responses, e.g.:

"No explanation, but I support the article! - a highly actual comment. by Gladkikh(15/07/20 14:45)" (http://litset.ru/publ/7-1-0-59553);

"Vyr, thank you for the topic and its extremely interesting and useful coverage)) - a comment by Klyouvik (14/07/20 19:13)" (http://litset.ru/publ/7-1-0-59553)

The above example reveals that a pompous master of rhetoric, an eye-catching fighter againsth "snobs and moralists" from science may cause a flow of positive feedback from the readers thus shaping up their outlook, knowledge of the languages in particular and science in general.

The following statistical analysis shows the following dependencies of the feedback to the site commentaries:

- maximal number of side responses to the article - 226;

- same minimal - 0;

- average for 100 publications, in succession - 3.71;

- maximal number of responses to the author's "Vyr" - 19;

- average feedback - 7.3 for all the author's publications.

Considering the above, even though the author in question never belongs to the top authors of the WWW source, their index is over the average in view of their random publications presented in succession.

This speaks of a sufficient interest drawn from the users of the site and the impact on their outlook and linguistic perception on the whole.

In general, WWW publications contain a plethora of highly doubtful recommendations, like "Quite a frequent error one may find in the reference materials of literary sites and also in the reviews of novice critics in treating an epithet as a trope. Part of the authors may probably claim that this purely professional issue has nothing to do with creative work. However, little care about such trifles may disclose an amateur who cares little about literature and poetry with their age-long features in favor or their own ambitions, and not in the public eyes, but in their own ones" (https://www.litprichal.ru/work/102824/) [4].

Quite evidently, referring an epithet to the category of tropes or to a separate category is a purely professional literary problem that reflects one of the opinions that exist in the scientific community $[9,12]$. This issue has popped up quite recently, while in the historical paradigm, since antiquity, the epithet has always been related to tropes. It is really hard to accept labeling as narcissists the authors like Cicero, Trediakovsky, and Soumarokov only because nowadays we classify tropes in a different way. The author also adds: "In fairness, it should be said that you may quite often come across the phenomenon of metaphorizing the epithet (golden ear), although it is primarily a highlighting figure never claiming to create a meaningful image, like tropes do".[ https://www.litprichal.ru/work/102824/]. This makes one think about at least a biased treatment of the notion of "metaphoric epithet", the essence of figures of speech and the mechanism of forming imagery in fiction. 
The above list of WWW sources reveals their predominantly "educational" nature. Regardless of their formally declared category, they are presented as something intentionally new, unknown to the reader, or just denouncing a certain false fact. It is noteworthy that this "new" or "true" is, in fact, not new or true at all. The reader is being misled about the fact or its interpretation. Structurally, a text of an "educating" article in the Internet contains one or a few assertions, never supported with objective data. Moreover, the proof system may be mentioned but never presented, e.g.: "according to the research" (where, which, source, etc are never mentioned in the text); "welscientists $\backslash$ Western experts/specialists have studied and discovered" (no results presented), "international experience shows" (why data presented is labeled international, who shares it, what research). Reasoning around such theses seems nothing but an effort to logically impact the reader's psychology instead of presenting evidence. More often, proof is based on a quasi-evident stream of arguments that intentionally have nothing to do with reality $[14,17,18]$.

You may also witness another typical aspect of non-objective quasi-scientific and quasieducating process. Now it's self-positioning the author as an expert in possession of certain specific knowledge. The trust in the data obtained normally grows, provided it is shared by a professional. This frame is often used by laymen in their works. Here, the WWW user is bound to accept as true the author's clam of being a professional, for it cannot be checked unless the reader is an expert in the field. Along with fakes and deliberate positioning of the presented theses as the truth, there grows the role of data, absolute and indistinguishable from accurate ones. To deal with this issue, the user has to undergo special training in the field of information technologies to be able to estimate, if not the accuracy of the data, then the source proper.

The authors of inaccurate publications widely use psychological influence on the reader. The key factor applied in making the reader believe that the presented facts are true is acting on their ego, emotions, personal preferences, fears. The example we presented above may serve here again: "...may disclose an amateur who cares little about literature and poetry with their age-long features in favor or their own ambitions". [4,23,32]

It is quite natural that the reader would choose to agree with the author's theses, because for them "literature and poetry with their age-long features" is of highest importance. Apart from some shallow psychological tricks, such authors make use of direct attacks on another author's personality. Aggressive behavior, low-grade vocabulary, direct humiliation and alienation of the opponent are frequent in quasi-professional publications. Their authors never admit other points of view. They actively oppose to "academism" and "misoneism" (in fact, opposing to objective data), demand "personal freedom".

Considering the above, the concept of knowledge proper gets washed away in the society developing indifference to the quality of information sources or even distrusting all of them $[13,25]$. Potentially, any source may equally contain true and false information. On the other hand, the falling status of information accuracy predetermines a carefree attitude to any information being made public. Multiple errors give birth to the lack of both inner needs in the accuracy of information and responsibility for its correct presentation.

We as the authors here believe that the society is ripe enough to deal with the issues of the objective knowledge of liberal arts and popular science promotion in mass media. We trust that the scientific society should develop a mechanism to help counteract the spread of inaccurate information in the virtual space of the Intermit, while popular-science promotion should be managed by the scientific society more intensively and in more popular and accessible form to be absorbed by a wider variety of those interested [15,19].. 


\section{Discussion}

As an example of an objective linguistic research we offer the method of pragmalinguistic experiment [21,22]. This approach to studying linguistic reality (discourse) suggests separation of certain structural and functional components (actualized modal-predicative groups, speech signals etc.), their statistical calculations, interpretation and further use to diagnose individual peculiarities of the speech behavior of the linguistic persona.

Mathematical simulation allows for forecasting a long-term effect of appealing function on the material object of social group. Such approach is quite widespread in science in general and linguistics in particular, and its objectivity is confirmed by repetition, consistency, and practical application of results that serve to improve the professional activities of experts in the field of social sciences and liberal arts. [16].

The level of response to a text is measured by means of questionnaires and tracking the receiver's reaction to a message $[30,33]$. Measuring units here are small predicate unities small syntactic groups (SSG) [ 20,21].

The specific features of this experiment arise from the object under study, targets, and matter of research. As a rule, the focus here falls on studying the pragmalinguistic characteristic features of the speech activity and behavior of the social group or even, at times, of a person [21]. The final aim of the research is determining the communicative and behavioral parameters reflected in speech. The material of research is speech discourse after being determined by the researcher in a chosen body of texts. Considering the final aim of research, to process a vast amount of material we make use of up-to-date software, like the one used for calculating and simulating dependencies in the Excel medium.

Studying and interpreting text bodies by their numerical patterns allows us to extract the vital, qualitative and functional patterns of the speech model out of the multitude of individual variants of its realization in direct communication.

The methods of mathematical simulation in pragmalinguistics permit to objectively describe some current language and speech phenomena and also to forecast their various states along a definite perspective.

The key confirmation to any suggested hypothesis arises from an experiment. One of a few desirable methods of experimental confirmation of the objectivity of a mathematical model is an experiment held based on the method of modified content analysis. The algorithm of this experiment lies in the maximal objectification of the data received and interpreted which allows for creating an objective model and detecting the true patterns of the event under study.

The first stage of the experiment lies in preparing the body of speech signals.

The second stage is picking out some textual material for analysis. Each text here is treated as a complete speech, a communicative and speech unity. The speech message senders are selected according to the unified stratification features.

The third stage features dividing blog texts into small syntactic groups (SSG) and analyzing the patterns of the usage of single-type speech signals in SSG which facilitates establishment of the typical features of the speech activity of the individual.

The next stage is processing the data:

1) Calculation of the frequency of the actualization of hidden intentions in per cent using the following formula:

$$
\mathrm{F} \text { plan }=(\text { E ssg plan }+ \text { E ssg plan }) \times 100 \%,
$$

where $\mathrm{F}$ plan is the frequency of the occurrence of the plan in \%, E - total SSG;

2) Deriving a mean speech-manner index (SMI) for each plane of a strategy under study. The mean speech-manner index is understood as a speech behavioral norm, typical of each group of authors; comparing individual author's indices to SMI results in a deviation in the 
behavior of the author from the conditional group standard. Calculations here are based on the following formula:

$$
\text { S plan }=(\text { F plan } \mathrm{A} 1+\ldots+\text { F plan } A n)
$$

where S plan is SMI, F plan A1 is the frequency of occurrence of the speech plane of the first author, F plane Ap is the frequency of occurrence of the given speech plane of the $n$-author; $\mathrm{n}$ is the number of authors under study (from 1 to 10 ).

3) Further on, we determine a permissible step of deviation from SMI and its trust interval which allow determining the absolute speech- manner indices (ASMI), relevant for diagnostics. Diagnosed are those ASMI which are higher or lower the permissible step of deviation from SMI (AS) and are not included in the trust interval. Trust interval is understood as the gap of meanings which includes permissible steps of deviation from SMI toward an increase or decrease in the values, so it shouldn't be regarded as diagnosing.

It terms of mathematical statistics, ASMI should be treated as discreet random values which, contrary to constant ones, change spasmodically. To give an explanation to the distribution of these values, we are bound to use the basic numerical characteristics of the random value.

The trust interval for the SMI mean value equals $\mathrm{SMI} \pm \mathrm{AS}$.

4) The final stage means interpretation of speech portraits considering the hidden strategies under study and the diagnostics of the authors' personal characteristics.

This approach has been used for the last 25 years for analyzing and revealing speech parameters which speaks for the reliability of scientific approach in studying linguistic data by means of pragmalinguistic methods and mathematical stimulation $[6,8,10,11,31]$.

\section{Conclusion}

To conclude, it should be indispensable to note that in between the virtual nonobjective understanding of linguistics and the present-day objective scientific research there exist students and laymen, interested in liberal arts. For an untrained recipient of WWW information the Internet offers a simple and easily accessible, though inaccurate, information that may misguide. The scientific methods of perception require special training, are hard to perceive and often may not be explained in simple words. Even though scientific learning requires special training, it never presents an objective picture of the world.

To make the contemporary student get acquainted with the objective world, the scientist needs to be a decent storyteller and interpreter, while contemporary science should be more widely popularized in the Internet.

\section{References}

1. A. Gunawardana, C. Meek, P. Xu, Microsoft Research, 1-8 (2011)

2. C.D. Manning, P. Raghavan, H. Schütze (Cambridge University Press, 2008)

3. R.Feldman, J. Sanger (Cambridge University Press, 2007)

4. Goys Gespräche http://www.welt.de/print/die_welt/hamburg/article13708759/Ich-habgern-alles-unter-Kontrolle.htm

5. Kraftwerk: Ralf Hütter im Interview (1991) http://www.musikexpress.de/ fundstuecke/article682309/kraftwerk-ralf-huetter-im-interview-1991.html

6. Radioeins http://www.rbb-online.de/kultur/beitrag/2015/01/kraftwerk-radioeinsexklusiv-interview-ralf-huetter.html 
7. Scorpions http://www.faz.net/aktuell/feuilleton/pop/scorpions-saenger-klaus-meine-imgespraech-12911774.html

8. D.O. Zhukov, S.A. Lesko, IEEE International Conference on Social Communication, 870-873 (2015)

9. N.G. Arkhipova, Word: Folklore-but-dialectological almanac 7, 85 (2009)

10. M.M. Bakhtin, Russian literature: From the theory of literature to the structure of the text: an anthology (Academia, Moscow, 1997)

11. E.V. Brysina, Bulletin of the Chelyabinsk state University 16(307), 25-31 (2013) http://www.lib.csu.ru/vch/307/vcsu13_16.pdf

12. N.-E. Buattura, L.S. Maikovskaya, Vestnik MGUKI http://files.msuc.org/Vestnik/2012-3/2012\%20-\%203\%20-\%20216.pdf

13. A. Vezhbitskaya, New in foreign linguistics Moscow VIII, 402-407 (1978)

14. V.V. Vinogradov (Nauka, Moscow, 1975)

15. S.Yu. Golovin (1998) http://vocabulary.ru / dictionary/25/word/yemocija

16. V.N. Egorova, Vestnik Nizhegorodskogo Universiteta 1(2), 360-361 (2012)

17. I.I. Eliseeva, M.M. Yuzbashev (Finance and statistics, Moscow, 2005)

18. E.P. Ilyin, Emotions and feelings (Piter, Saint Petersburg, 2001)

19. A.N. Leontiev (Moscow state publishing House UN-TA, Moscow, 1971)

20. N.V. Lukina (2011) http://cheloveknauka.com/smyslovaya-struktura-metateksta.

21. M.V. Lapon, Big Russian encyclopedia (Drofa, Moscow, 1998)

22. G.G. Matveeva (St. Petersburg, 1993) http://dlib.rsl.ru/viewer/01000320357\#?page=1

23. G.G. Matveeva, G.I.Myasishchev, Yu.P. Khoroshevskaya, Bulletin of the Chelyabinsk state University 10(432), 88-92 (2019)

24. T.S. Medvedeva, Bulletin of the Udmurt University 5(2), 49 (2011)

25. Yu.I. Nastusenko, Student scientific forum: Mat-ly VII international. student of the electronic scientific conference http://www.scienceforum.ru/2015/1204/16067

26. N.P. Perfileva (2014) http://psycholinguistik.narod.rU/olderfiles/6/Perfileva_ADD.pdf

27. E.A. Sidorenko, G.G. Matveeva, Bulletin of Pyatigorsk state University 1, 179-184 (2019)

28. E.A. Sidorenko, G.G. Matveeva, News of the southern Federal University. Philological science 4, $72-79$ (2019)

29. D.B. Sundueva, Bulletin of the Chita state University 4(71), 63-69 (2011)

30. E.D. Chomskaya, N.Ya. Batova (MSU publishing House, Moscow, 1992)

31. A.K. Halliday, Linguostylistics $I X$ (Progress, M., 1980)

32. E.V. Shirina, Speech activity. Inter university collection of scientific papers (Taganrog, 2002)

33. R.O. Yakobson (Progress, Moscow, 1985) 